\title{
Ebola and the Kidney
}

\author{
Weeraporn Srisung MD
}

The Ebola virus disease (EVD) re-emergence in March 2014 has caused global alarm among the public as well as with health care providers. Infected patients usually present with nonspecific symptoms, such as vomiting, diarrhea, fever, and visible hemorrhage. Mortality rates in previous outbreaks have varied from $25-90 \%$. EVD is transmitted by direct contact with the blood or other bodily fluids of infected patients and with contaminated surfaces and materials. It results in multiorgan failure, with acute kidney injury (AKI) present in many cases. ${ }^{2}$

Acute kidney injury in EVD is likely multifactorial. EVD usually causes a systemic inflammatory response syndrome and capillary leakage, similar to mechanisms that occur in sepsis. These events, together with massive fluid loss from vomiting and severe diarrhea, can lead to pre-renal azotemia. ${ }^{2}$ Acute tubular necrosis (ATN) is another type of renal injury that occurs in EVD. This can be due to multiple agents or clinical events. Renal ischemia can follow pre-renal azotemia, resulting in ATN. Cytokine storm, superinfection with bacterial pathogens or malaria, and therapeutic agents, including antibiotics, are other factors contributing to ATN ${ }^{3}$ Studies also suggest that the Ebola virus infects renal tubular cells; therefore, direct injury to renal tubules is a possible cause of ATN. Additionally, the clotting abnormalities and factors related to hemorrhagic fever can predispose patients to disseminated intravascular coagulation. ${ }^{2.3}$ Retroperitoneal hemorrhage and abdominal compartment syndrome are complications of EVD, and with the presence of either of these conditions, AKI can develop or become worse. ${ }^{3}$

Corresponding author: Weeraporn Srisung MD Contact Information: Weeraporn.srisung @ttuhsc.edu DOI: 10.12746/swrccc2015.0309.111
Dialysis is often used in patients with EVD. A clinical case report and clinical practice guidelines for renal replacement therapy in the acute phase of EVD have recently been published. ${ }^{4}$ The Centers for Disease Control and Prevention (CDC) also provided recommendations for the safe performance of acute hemodialysis in EVD patients. ${ }^{5}$ Indications for dialysis in Ebola-infected patients are similar to those in other patients with AKI. EVD has a substantial risk of nosocomial infection, with the viral levels reaching 1010 copies/mL of blood during acute infection. Moreover, patients with EVD for whom dialysis is necessary contain the highest degree of viremia and thus the highest risk of transmission. Therefore, dialysis should be initiated only when health care providers can be appropriately protected from virus exposure. Additionally, physicians should consider health care providers' safety when deciding whether or not to perform any invasive or non-invasive interventions. Limiting laboratory or radiological studies may be useful to minimize the transmission risk to health care professionals. ${ }^{2}$ The CDC recommends the use of Personal Protective Equipment, and all health care workers including dialysis providers must adhere to these guidelines. $^{6}$

Dialysis providers should carefully consider center-specific expertise when modes of therapy and equipment are chosen, since employing new procedures or methods of dialysis can increase errors. ${ }^{3}$ As in other AKI patients, continuous renal replacement therapy (CRRT) generally offers advantages over intermittent hemodialysis (IHD) in hemodynamically unstable patients. Restricting the patient to a single CRRT machine throughout the hospitalization and decreasing the frequency of filter change of the CRRT system should also be considered. Point-of-care laboratory testing is used in many centers to minimize the frequency of the clinical laboratory to handle specimens. Empiric supplementation of some electrolytes may be considered in cases in which point-of-care 
testing cannot offer monitoring of certain electrolytes, such as magnesium and phosphorus. Once the patient improves from the acute phase and no longer needs hemodynamic support, IHD may be required instead of CRRT. ${ }^{2}$

Vascular access for dialysis should be performed by experienced healthcare professionals. Ultrasound guidance should be used, and the equipment, including the ultrasound machine, should remain in the room. ${ }^{2}$ The right internal jugular position is the preferred site for vascular access. ${ }^{4}$ In patients with end-stage renal disease undergoing maintenance hemodialysis who are infected with the Ebola virus, placement of a new dialysis catheter may reduce the risk of exposure from a fistula or graft, although the placement can be challenging since most of these patients have had previous vascular injury. For those who require maintenance peritoneal dialysis, placement of a temporary HD catheter should be considered since the virulence of peritoneal ultrafiltrate is unknown. ${ }^{2}$

Theoretically, dialysis effluent should be sterile since the size of the Ebola virus is larger than the pore size. However, blood leaks can occur, so all waste products from dialysis should be assumed to be infectious. ${ }^{2,4}$ The CDC suggests that disinfection of dialysis effluent is not required, and it can be safely disposed into municipal wastewater streams, such as the toilet, sink, or wall drain. However, confirmation with local or state health departments and local wastewater management is required. ${ }^{3}$

Author Affiliation: Weeraporn Srisung is a resident in internal medicine in the Department of Internal Medicine at Texas Tech University Health Sciences Center in Lubbock, TX.

Received: $11 / 30 / 2014$

Accepted: 12/11/2014

Reviewers: Vaqar Ahmed MD

Published electronically: 01/15/2015

Conflict of Interest Disclosures: none

\section{REFERENCES}

1. World Health Organization. (September, 2014). Ebola virus disease. Retrieved November 30, 2014 from http://www.who.int/ mediacentre/factsheets/fs103/en/.

2. American Society of Nephrology. (October 24, 2014). Frequently asked questions (FAQs) regarding Ebola virus disease and dialysis. Retrieved November 30, 2014 from http://www. asn-online.org/news/2014/ASN FAQs Ebola Virus Disease and_Dialysis.pdf.

3. Connor, MJ, Franch, HA, Geiger, H, Faubel, S, Evans, NG. (November 14, 2014). Ebola and dialysis: Resources for Nephrology Health Professionals. Symposia conducted at American Society of Nephrology Kidney Week 2014, Philadelphia, PA.

4. Connor MJ, Jr., Kraft C, Mehta AK, Varkey JB, Lyon GM, Crozier I, et al. Successful delivery of RRT in Ebola virus disease. J Am Soc Neph 2014 Nov 14. pii: ASN.2014111057. [Epub ahead of print].

5. Centers for Disease Control and Prevention. (n.d.). Recommendations for safely performing acute hemodialysis in patients with Ebola virus disease in U.S. hospitals. Retrieved November 30, 2014 from http://www.cdc.gov/vhf/ebola/hcp/guidance-dialysis.html.

6. Centers for Disease Control and Prevention. (October 20, 2014). Guidance on personal protective equipment to be used by healthcare workers during management of patients with Ebola virus disease in U.S. hospitals, including procedures for putting on (donning) and removing (doffing). Retrieved November 30 , 2014 from http://www.cdc.gov/vhf/ebola/hcp/procedures-forppe.html. 